\title{
SAMW-Richtlinien «Feststellung des Todes mit Bezug auf Organtransplantationen» umfassend überarbeitet
}

Zur Feststellung des Todes verweist die Verordnung zum Transplantationsgesetz auf die SAMW-Richtlinien «Feststellung des Todes mit Bezug auf Organtransplantationen» aus dem Jahre 2005. Damit unterstellt der Gesetzgeber nicht die Definition des Todes, jedoch die Bestimmungen, wie dieser lege artis festzustellen ist, dem Stand der medizinischen Wissenschaft. Weil die bisherigen Richtlinien in der Praxis $\mathrm{zu}$ Interpretationsschwierigkeiten führten, hat eine Subkommission unter dem Vorsitz von Prof. Jürg Steiger aus Basel diese umfassend überarbeitet. Ende Mai 2011 hat der SAMW-Senat die revidierten Richtlinien verabschiedet; sie treten am 1. September 2011 in Kraft.

Die neuen Richtlinien sehen namentlich in folgenden Bereichen Änderungen vor:

- Sofern die Ursache für den Funktionsausfall des Gehirns eindeutig feststeht, braucht es für die Feststellung des Todes nicht mehr zwei, sondern nur noch eine klinische Untersuchung; diese muss jedoch durch zwei Ärzte vorgenommen und die Diagnose des Hirntodes von beiden bestätigt werden (Vier-Augen-Prinzip).

- Wenn feststeht, dass die Prognose bei einem Patienten aussichtslos ist, darf die Möglichkeit einer «Organspende» mit den Angehörigen besprochen werden.

- Unter definierten Voraussetzungen können vorbereitende medizinische Massnahmen im Hinblick auf eine Organtransplantation durchgeführt werden; wenn der potentielle Spender diesen nicht ausdrücklich zugestimmt hat, ist auf seinen mutmasslichen Willen Bezug zu nehmen.

Von Mitte Februar bis Ende März 2011 stand der Entwurf der neuen Richtlinien zur Vernehmlassung. Es trafen über 60 Stellungnahmen ein; praktisch alle haben sowohl die Notwendigkeit der Revision als auch die zentralen Revisionspunkte befürwortet.

In einzelnen Stellungnahmen wurde der Verzicht

Korrespondenz:

lic. iur. Michelle Salathé Schweizerische Akademie der Medizinischen Wissenschaften SAMW

Petersplatz 13

CH-4051 Basel

mail@samw.ch
Literatur gefordert. In der definitiven Fassung der Richtlinien wurde nun eine gemeinsame Feststellung des Todes durch zwei Ärzte (Vier-Augen-Prinzip) eingeführt.

Als erklärungsbedürftig wurden vielfach auch die unterschiedlichen Vorgaben bei den Kleinkindern beurteilt: einerseits der Verzicht auf Organentnahmen bei Neugeborenen und andererseits das Festhalten an zwei klinischen Untersuchungen mit einem Zeitintervall von $24 \mathrm{~h}$ bei Säuglingen unter einem Jahr. Bis auf weiteres wird an dieser Regelung festgehalten, weil es zur Verlässlichkeit der Feststellung des Hirntodes beim Neugeborenen bislang weniger wissenschaftliche Evidenz gibt als beim Erwachsenen. Zusätzlich sprechen auch medizinische Gründe wie z. B. die hohe Nachgiebigkeit der Schädelstrukturen bei erhöhtem Hirndruck dafür.

Mit Vorbehalt wurde in einzelnen Stellungnahmen auch die liberale Interpretation des Transplantationsgesetzes (TxG) bezüglich der «vorbereitenden medizinischen Massnahmen» aufgenommen, wenn der mutmassliche Wille des potentiellen Spenders nicht bekannt ist. Die Subkommission hatte sich dabei auf ein vom BAG veranlasstes Rechtsgutachten zum TxG gestützt. Auch diesen Bedenken wurde in der definitiven Fassung der Richtlinien Rechnung getragen: Vorbereitende Massnahmen sollen zulässig sein, wenn der potentielle Spender ihnen mutmasslich zugestimmt hätte. Die vom BAG im April 2011 neu gestaltete Bevölkerungsinformation zur Organtransplantation (inkl. Spendekartenbroschüre) unterstützt diese Stossrichtung. Neu werden auch die für die Organspende notwendigen vorbereitenden medizinischen Massnahmen beschrieben. Die aktuell in Vernehmlassung stehende Teilrevision des TxG schlägt zudem eine Anpassung der Bestimmungen zu den vorbereitenden Massnahmen vor.

Voraussichtlich zeitgleich mit der Inkraftsetzung der Richtlinien am 1. September 2011 wird der Verweis auf die SAMW-Richtlinien im Anhang der Verordnung zum Transplantationsgesetz aktualisiert.

Die Richtlinien können von der Website der SAMW heruntergeladen werden (www.samw.ch unter: Ethik/Richtlinien) oder als Broschüre beim Generalsekretariat der SAMW bestellt werden. 


\section{Feststellung des Todes mit Bezug auf Organtransplantationen}

\section{Präambel}

Seit dem 1. Juli 2007 sind die rechtlichen Voraussetzungen für Organtransplantationen auf gesamtschweizerischer Ebene im Bundesgesetz über die Transplantation von Organen, Geweben und Zellen (Transplantationsgesetz) [1] festgehalten. In der Frage des Todeskriteriums stützt sich das Gesetz auf die neurologische Definition des Todes, wonach der Mensch tot ist, wenn sämtliche Funktionen seines Hirns, einschliesslich des Hirnstamms, irreversibel ausgefallen sind. Zur Feststellung des Todes verweist die Verordnung zum Transplantationsgesetz auf die SAMW-Richtlinien «Feststellung des Todes mit Bezug auf Organtransplantationen». Damit unterstellt der Gesetzgeber nicht die Definition des Todes, jedoch die Bestimmungen, wie er lege artis festzustellen ist, dem Stand der medizinischen Wissenschaft. Nachdem der Tod festgestellt ist, dürfen Organe entnommen werden, wenn eine Einwilligung des allfälligen Spenders oder subsidiär eine stellvertretende Einwilligung von berechtigten Dritten vorhanden ist (sog. erweiterte Zustimmungslösung).

Die Definition des Todes beinhaltet neben biologischen und medizinischen auch rechtliche und ethische Aspekte. Sie enthält nicht nur Aussagen über pathophysiologische Zustände, die mit dem Tod assoziiert sind, sondern impliziert immer auch ein spezifisches Verständnis von Leben und Tod. Sie drückt insbesondere auch aus, welche Handlungen an Verstorbenen vorgenommen werden dürfen. Die Abklärung des Patientenwillens, die Behandlung eines Organspenders bis zur Feststellung des Todes, die Durchführung von organerhaltenden medizinischen Massnahmen bis zur Entnahme der Organe und der Umgang mit den Angehörigen stellen für das Behandlungsteam eine grosse Herausforderung und Belastung dar. Aus ethischer und rechtlicher Perspektive ist es zwingend, den Tod auf zuverlässige und sichere Weise zu diagnostizieren, dem Willen der verstorbenen Person Rechnung zu tragen und die Angehörigen in dieser schwierigen Phase zu unterstützen. Diesem Ziel dienen die vorliegenden Richtlinien. Einerseits bestimmen sie die klinischen Zeichen und technischen Zusatzuntersuchungen, die bei der Feststellung des Todes $\mathrm{zu}$ berücksichtigen sind; andererseits beschreiben sie die Prozesse bis zur Organentnahme und die Verantwortung der involvierten Ärzte [2] und weiterer Fachpersonen. Sie äussern sich auch zur
Organentnahme nach einem irreversiblen Kreislaufstillstand (sog. Non-Heart-Beating Donation) und den mit dieser Situation verbundenen spezifischen ethischen Fragestellungen.

Die nachfolgenden Richtlinien stützen sich sowohl auf das Transplantationsgesetz (nachfolgend: TxG) als auch auf ein Rechtsgutachten zu Fragen im Zusammenhang mit der Organentnahme bei verstorbenen Personen und vorbereitenden medizinischen Massnahmen [3].

\section{Richtlinien}

\section{Todeskriterium [4]}

Der Mensch ist tot, wenn die Funktionen seines Gehirns einschliesslich des Hirnstamms irreversibel ausgefallen sind [5].

Durch den irreversiblen Ausfall sämtlicher Funktionen des Gehirns verliert ein Mensch das Steuerungsorgan des gesamten Organismus endgültig. Nachfolgend sterben alle Organe, Gewebe und Zellen unabwendbar ab.

Der Tod kann durch folgende Ursachen eintreten:

- durch den irreversiblen Funktionsausfall des Hirns einschliesslich des Hirnstamms infolge primärer Hirnschädigung oder -erkrankung;

- durch anhaltenden Kreislaufstillstand, der die Durchblutung des Gehirns so lange reduziert oder unterbricht, bis der irreversible Funktionsausfall von Hirn und Hirnstamm und damit der Tod eingetreten ist (Tod nach Kreislaufstillstand).

\section{Feststellung des Todes}

\subsection{Tod infolge primärer Hirnschädigung}

Die Feststellung des Todes erfolgt durch eine klinische Untersuchung, welche die folgenden sieben klinischen Zeichen nachweisen muss; diese müssen kumulativ vorhanden sein:

1. Koma;

2. Beidseits weite, auf Licht nicht reagierende Pupillen;

3. Fehlen der okulozephalen (zervikookulären und vestibulookulären) Reflexe;

4. Fehlen der Kornealreflexe;

5. Fehlen zerebraler Reaktionen auf schmerzhafte Reize;

6. Fehlen des Husten- und Schluckreflexes;

7. Fehlen der Spontanatmung (Apnoetest). 
Die klinische Untersuchung erfolgt gemeinsam durch zwei Ärzte (Vier-Augen-Prinzip); einer der Untersucher darf nicht direkt in die Betreuung des Patienten involviert sein.

Beide den Tod diagnostizierenden Ärzte müssen eine entsprechende Qualifikation aufweisen (siehe Kap. 2.4.).

Liegt ein klarer Grund für den Funktionsausfall des Gehirns vor und sind die Umstände und Modalitäten gemäss Anhang [6] berücksichtigt, beschränkt sich die Diagnostik auf die klinischen Zeichen [7].

Ist der Funktionsausfall des Gehirns durch die in der Bildgebung nachgewiesene strukturelle Schädigung nicht hinreichend erklärt, können potentiell reversible Faktoren als Mitursache nicht ausgeschlossen oder können die Funktionen der Hirnnerven klinisch nicht untersucht werden, muss die Abwesenheit der zerebralen Durchblutung mit einer geeigneten $\mathrm{Zu}$ satzuntersuchung nachgewiesen werden.

Dafür können die nachfolgenden Verfahren angewendet werden:

- transkranielle Doppler- oder Farbduplexsonographie;

- Computertomographische Angiographie (CTA);

- intraarterielle digitale Subtraktionsangiographie (IA-DSA);

- Magnetresonanztomographie und -angiographie. Die Anforderungen an die einzelnen technischen Verfahren sind im Anhang [8] festgehalten.

\subsection{Tod nach anhaltendem Kreislaufstillstand} Der Tod bei anhaltendem Kreislaufstillstand ist gemäss Transplantationsgesetz ebenfalls durch den irreversiblen Ausfall der Funktionen von Gehirn und Hirnstamm definiert. Der Tod tritt dabei durch die anhaltende Unterbrechung der Durchblutung des Gehirns infolge eines Kreislaufstillstands ein.

Nach Feststellung des Kreislaufstillstands (fehlende Herzaktivität) mittels transthorakaler Echokardiographie (TTE) im 4-Kammerblick oder in der subxiphoidalen Einstellung und Verstreichen einer Wartezeit von mindestens 10 Minuten ohne Durchführung von Reanimationsmassnahmen werden die nachfolgenden klinischen Zeichen geprüft; diese müssen kumulativ vorhanden sein:

1. Koma;

2. Beidseits weite, auf Licht nicht reagierende Pupillen;

3. Fehlen der okulozephalen (zervikookulären und vestibulookulären) Reflexe;

4. Fehlen der Kornealreflexe;

5. Fehlen zerebraler Reaktionen auf schmerzhafte Reize;

6. Fehlen des Husten- und Schluckreflexes;

7. Fehlen der Spontanatmung.

Die klinische Untersuchung erfolgt gemeinsam durch zwei dafür qualifizierte Ärzte (Vier-Augen-Prinzip); einer der Untersucher darf nicht direkt in die Betreuung des Patienten involviert sein. Zusatzuntersuchungen sind nicht notwendig, da der dokumentierte Kreislaufstillstand mittels TTE über einen Zeitraum von 10 Minuten eine genügende Hirndurchblutung ausschliesst [9].

\subsection{Tod bei Kindern}

Bei Kindern, die älter als ein Jahr sind, sind die Regeln gemäss Kapitel 2.1. und 2.2. anwendbar.

Bei Säuglingen [10] jenseits der Neonatalperiode, erfolgt die Feststellung des Todes - sofern die Ursache des zerebralen Funktionsausfalls bekannt ist - durch zwei klinische Untersuchungen (inklusive Apnoetest) mit einem Beobachtungsintervall von 24 Stunden [11]. Ist der Funktionsausfall des Gehirns durch die in der Bildgebung nachgewiesene strukturelle Schädigung nicht hinreichend erklärt, können potentiell reversible Faktoren als Mitursache nicht ausgeschlossen oder können die Funktionen der Hirnnerven klinisch nicht untersucht werden, muss die Abwesenheit der zerebralen Durchblutung nach der zweiten klinischen Untersuchung mit einer geeigneten Zusatzuntersuchung nachgewiesen werden.

Bei Neugeborenen [12] ist aufgrund von ethischen und medizinischen Überlegungen auf die Entnahme von Organen zum Zweck der Transplantation zu verzichten [13].

\subsection{Fachliche Voraussetzungen}

Die klinische Beurteilung muss durch Ärzte mit Weiterbildung und Erfahrung im Bereich Hirntoddiagnostik erfolgen [14].

Für die klinische Feststellung des Todes bei Kindern ist eine Weiterbildung in pädiatrischer Intensivmedizin oder Neuropädiatrie erforderlich.

Die Durchführung der Zusatzuntersuchung muss durch einen Facharzt mit der jeweils spezifischen Qualifikation erfolgen.

\subsection{Unabhängigkeit}

Um Interessenkonflikte zu vermeiden, müssen die Prozesse der Organentnahme und -transplantation streng getrennt werden.

Gemäss Artikel 11 TxG dürfen Ärzte, die den Tod im Hinblick auf eine Organentnahme feststellen:

a. weder an der Entnahme noch an der Transplantation von Organen, Geweben, Zellen mitwirken;

b. noch den Weisungen einer ärztlichen Fachperson unterstehen, die an solchen Massnahmen beteiligt ist.

In der Intensivmedizin stellt sich die Frage der Unabhängigkeit in besonderem Mass. Ein Interessenkonflikt kann bereits bei der Frage des Abbruchs von Massnahmen respektive deren Weiterführung entstehen. Es ist nicht ausgeschlossen, dass mögliche Empfänger auf derselben Intensivstation wie der potentielle Spender hospitalisiert sind. Diesem Umstand wird durch den Zuzug eines unabhängigen Zweitarztes bei der Feststellung des Todes Rechnung getragen (Vier-Augen-Prinzip).

\subsection{Dokumentation}

Die klinischen Befunde, allfällig durchgeführte Zusatz- 
untersuchungen sowie Gespräche betreffend Einwilligung sind schriftlich festzuhalten. Dafür stehen die Protokolle im Anhang zur Verfügung; diese können spitalintern ergänzt werden.

\section{Abklärung des Patientenwillens}

Die nachfolgend geschilderten Schritte gelten sowohl für die Organentnahme beim Tod infolge primärer Hirnschädigung als auch für die Organentnahme beim Tod nach Kreislaufstillstand (NHBD). Beim Gespräch über eine allfällige Organentnahme nach einem Kreislaufstillstand sind jedoch zusätzliche Aspekte zu beachten (vgl. Kapitel 5.).

\subsection{Gespräch über die Organspende und die Durchführung von organerhaltenden Massnahmen}

Patienten, die als Organspender in Frage kommen, sind in der Regel nicht urteilsfähig. Die Möglichkeit einer Organspende ist deshalb mit dem gesetzlichen Vertreter, respektive den Angehörigen, anzusprechen, falls der Patient als Organspender in Frage kommt. Dies verlangt ein hohes Mass an Einfühlungsvermögen und Rücksichtnahme. Wenn die Möglichkeit besteht, das Thema frühzeitig anzusprechen, ist dies sinnvoll, weil die Angehörigen dann länger Zeit haben, sich mit der Frage auseinanderzusetzen. Die Besprechung des Abbruchs der lebenserhaltenden Therapien (Änderung des Behandlungsziels) muss separat vor der Aufklärung über die Organspende und die dafür erforderlichen medizinischen Massnahmen erfolgen. Der Entscheid, die lebenserhaltende Therapie abzubrechen, darf nicht durch die Möglichkeit einer Organspende beeinflusst werden.

Ist der Tod unter voller Therapie eingetreten, sind die Gespräche entsprechend anzupassen. Die Mitteilung des Todes soll nach Möglichkeit getrennt von der Thematisierung der Organspende erfolgen.

Bei urteilsfähigen Patienten, die eine aussichtslose Prognose haben, sollte an die Möglichkeit der Organspende gedacht und das Thema aufgenommen werden, sofern keine offensichtlichen Gründe dagegen sprechen. Ist ein Patient bereit, seine Organe für eine Spende zur Verfügung zu stellen, muss er auch über die Notwendigkeit der Durchführung von organerhaltenden medizinischen Massnahmen informiert werden.

Im Gespräch über die Möglichkeit der Organspende muss der Respekt gegenüber dem Patienten und den Angehörigen zum Ausdruck kommen. Das Gespräch soll in einer ruhigen Umgebung und möglichst ohne Zeitdruck stattfinden.

Wichtig ist, dass der behandelnde Arzt diese Gespräche führt und Kontinuität gewährleistet ist.

Zusammenfassend muss das Gespräch zur Abklärung des Spenderwillens folgende Anforderungen erfüllen:

- vom Inhalt her: umfassende und verständliche Information über den gesundheitlichen Zustand und die Prognose des Patienten (Wahrscheinlich- keit des Todeseintritts), Abbruch der lebenserhaltenden Massnahmen, Inhalt und Umfang der organerhaltenden Massnahmen sowie deren Zweck und Auswirkungen, Feststellung des Todes, Ablauf einer allfälligen Organentnahme und Vorgehen nach erfolgter Organentnahme;

- von den Rahmenbedingungen her: ruhige Atmosphäre, Empathie und Wahrnehmung der Angehörigen; genügend Zeit zur Erklärung der Situation, Raum für Fragen und Anliegen, Angebot von zusätzlichen Gesprächen durch geeignete Fachpersonen, die möglichst über den gesamten Zeitraum zur Verfügung stehen.

\subsection{Einwilligung zur Organentnahme und zur Durchführung von organerhaltenden medizinischen Massnahmen}

Im Idealfall hat sich der potentielle Organspender vorgängig, z.B. mit einer Spendekarte, zur Organspende sowie zur Durchführung der organerhaltenden Massnahmen geäussert. Häufig ist dies jedoch nicht der Fall. In dieser Situation muss der Wille des Patienten abgeklärt werden. Dabei muss geprüft werden, ob Anhaltspunkte dafür vorliegen, dass der Patient einer Organentnahme und der Durchführung der organerhaltenden Massnahmen zugestimmt oder diese abgelehnt hat bzw. hätte. Die nächsten Angehörigen spielen bei dieser Abklärung eine zentrale Rolle.

Personen, die anstelle des Patienten entscheiden, haben dessen mutmasslichen Willen zu beachten; dieser hat Vorrang vor ihren eigenen Präferenzen.

a) Entnahme der Organe zur Transplantation

Hat der potentielle Spender sich nicht selbst zur Organspende geäussert, entscheiden die nächsten Angehörigen an seiner Stelle. Dazu berechtigt sind Personen gemäss nachfolgender Reihenfolge: 1 . Ehepartner oder eingetragene Partner, 2. Kinder, Eltern und Geschwister, 3. Grosseltern und Grosskinder und 4. andere nahestehende Personen. Die Entnahme von Organen ist unzulässig, wenn die vertretungsberechtigten Personen sie ablehnen. Dies trifft auch $\mathrm{zu}$, wenn ein Patient keine Person hat, die ihn vertreten kann, oder wenn diese nicht rechtzeitig kontaktiert werden kann [15].

b) Durchführung von organerhaltenden Massnahmen Ist ein gesetzlicher Vertreter [16] vorhanden oder hat der Patient die Vertretung einer Person seines Vertrauens übertragen, so entscheiden diese über Durchführung von organerhaltenden Massnahmen. Hat der Patient keinen gesetzlichen Vertreter, können - mit Zustimmung der Angehörigen - organerhaltende Massnahmen durchgeführt werden, wenn dies dem mutmasslichen Willen des Patienten entspricht [17]. Stellt sich heraus, dass der Patient der Durchführung der organerhaltenden Massnahmen vermutlich nicht zugestimmt hätte oder können die Angehörigen keine Auskunft darüber geben, was sich der Patient gewünscht hätte, muss auf die Durchführung von organerhaltenden Massnahmen verzichtet werden. 


\subsection{Spezifische Aspekte bei Kindern}

Die Entscheidungsprozesse bei Kindern unterscheiden sich bezüglich des Vorgehens bei volljährigen Personen lediglich dadurch, dass in dieser Situation immer gesetzliche Vertreter (in der Regel die Eltern) vorhanden sind, welche stellvertretend entscheiden. Im Übrigen gelten die Regeln gemäss Kap. 3.1. und 3.2. Sind die Eltern unterschiedlicher Auffassung und kann keine Einigung gefunden werden, ist von einer Organentnahme abzusehen.

\section{Organentnahme nach Tod infolge primärer Hirnschädigung}

Wenn bei einem Patienten mit primärer Hirnschädigung die Prognose aussichtslos ist, ändert sich das Behandlungsziel. Im Vordergrund steht nicht mehr die Lebenserhaltung, sondern die palliative Betreuung. Falls in dieser Situation keine offensichtlichen medizinischen Gründe [18] gegen eine Organspende vorliegen, kommt der Patient prinzipiell als Organspender in Frage und sein Wille im Hinblick auf eine Spende muss eruiert werden (vgl. dazu Kapitel 3.1. und 3.2.). Liegt eine Einwilligung zur Organspende vor, steht das behandelnde Team vor der Herausforderung, den Patienten in Würde sterben zu lassen, die Bedürfnisse der Angehörigen zu respektieren, gleichzeitig aber die transplantierbaren Organe durch spezifische medizinische Massnahmen in optimalem Zustand zu erhalten.

\subsection{Medizinische Massnahmen vor dem Tod} Bei den medizinischen Massnahmen ist zu unterscheiden zwischen Massnahmen, die der Organerhaltung dienen, und solchen, die eine Abklärung der Spendetauglichkeit zum Ziel haben.

Massnahmen zur Organerhaltung [19] sind Voraussetzung dafür, dass eine Organspende überhaupt stattfinden kann; sie sind entscheidend für den Erfolg einer Transplantation. In der Regel handelt es sich um die Fortführung von bereits begonnenen Therapien (Weiterführung der künstlichen Beatmung, Verabreichung von Medikamenten und Lösungen zur Erhaltung der Kreislauffunktion), Laboranalysen zur Steuerung der Behandlung sowie Hormonsubstitutionen zur Aufrechterhaltung des «Inneren Milieus». Nach der Änderung des Behandlungsziels sind diese nicht mehr im therapeutischen Interesse des Patienten, sondern werden zur Organerhaltung weitergeführt. Bei der Entscheidung, ob eine Massnahme zur Anwendung kommt oder nicht, müssen die individuelle Situation des Patienten berücksichtigt und die Risiken abgewogen werden. Diese medizinischen Massnahmen sind auf zwei Tage zu begrenzen.

Bei den Massnahmen zur Abklärung der Spendetauglichkeit handelt es sich primär um serologische und immunologische Analysen [20].

\subsection{Medizinische Massnahmen nach festge- stelltem Tod}

Nach festgestelltem Tod sind medizinische Massnah- men zur Erhaltung der Organe sowie Massnahmen zur Abklärung der Spendereignung während längstens 72 Stunden erlaubt [21].

\section{Organentnahme bei Tod nach Kreislaufstillstand}

\subsection{Maastricht-Klassifikation}

Grundsätzlich unterscheidet die Maastricht-Klassifikation folgende Situationen:

a) Tod bereits eingetreten bei Ankunft im Spital (Maastricht, Kategorie 1)

b) Tod nach erfolgloser Reanimation (Maastricht, Kategorie 2)

c) Tod nach Abbruch von lebenserhaltenden Massnahmen (Maastricht, Kategorie 3)

d) Kreislaufstillstand bei vorgängigem Tod infolge primärer Hirnschädigung (Maastricht, Kategorie 4) Für alle Kategorien gilt, dass die Organentnahme oder die Perfusion der Organe mit Konservierungslösung möglichst rasch nach der Todesfeststellung erfolgen muss, um die Zeit der organschädigenden warmen Ischämie möglichst kurz zu halten. Bei den Maastricht-Kategorien 1 und 2 sind die Angehörigen in der Regel nicht auf den Tod vorbereitet und müssen sich unter Zeitdruck mit der Frage der Organtransplantation auseinandersetzen. Dies ist eine äusserst belastende Situation.

a) Maastricht-Kategorie 1

Bei Organspendern der Kategorie 1 wurde der Tod bereits vor oder unmittelbar bei Ankunft im Spital festgestellt. Liegt eine Zustimmung zur Organspende vor, kann eine Organentnahme stattfinden. Liegt keine Zustimmung vor, können vorbereitende Massnahmen zur Organentnahme (insbesondere das Legen eines Doppelballonkatheters zur Perfusion der Organe mit kalter Konservierungslösung) vorgenommen werden, bis die Angehörigen befragt werden können (vgl. Kap. 5.3.).

b) Maastricht-Kategorie 2

Bei Organspendern der Maastricht-Kategorie 2 erfolgt die Organentnahme nach erfolgloser Reanimation. Weil bei einer kardiopulmonalen Reanimation ein reduzierter Kreislauf erhalten bleibt, darf die Feststellung des Todes erst nach Erfolglosigkeit der Reanimation und einer anschliessenden 10-minütigen Beobachtung des ununterbrochenen Herzstillstandes mit totalem Kreislaufausfall erfolgen (Normothermie [22] vorausgesetzt).

Erfolglose Reanimation bedeutet, dass trotz lege artis durchgeführter Wiederbelebungsversuche über mindestens 20 Minuten keine Rückkehr der Herzaktion mit spontanem Kreislauf erreicht wurde und der Patient die unter «klinische Zeichen» aufgeführten Befunde aufweist. Tritt unter der Reanimation vorübergehend wieder eine spontane, kreislaufwirksame Herzaktion auf, beginnt die 20-minütige Reanimationsfrist nach dem Ende dieser Herzaktion wieder neu.

c) Maastricht-Kategorie 3

Bei Organspendern der Maastricht-Kategorie 3 wird 
eine Organentnahme vorgenommen, nachdem der Patient infolge eines Abbruchs der lebenserhaltenden Therapie bei Erkrankung mit aussichtsloser Prognose verstorben ist. Diese Patienten haben eine normale Herztätigkeit, solange die lebenserhaltenden Massnahmen (insbesondere die künstliche Beatmung) weitergeführt werden. Nachdem diese Massnahmen abgebrochen werden, verstirbt der Patient infolge eines Herzstillstands. Sein Tod wird gemäss Kap. 2.2. festgestellt. Die Zeit zwischen Feststellung des Todes und der Organentnahme sollte so kurz wie möglich sein.

\section{d) Maastricht-Kategorie 4}

Bei Organspendern der Maastricht-Kategorie 4 tritt der Kreislaufstillstand unerwartet, nach Feststellung des Todes infolge primärer Hirnschädigung bei den Vorbereitungen zur Organentnahme auf. In dieser Situation stehen folgende Möglichkeiten zur Auswahl:

- Wiederherstellung der Funktion des Kreislaufs;

- rascher Transport in den Operationssaal;

- das Einlegen von Perfusionssonden (in der Regel Gillotsonde) zur Organpräservation;

- Verzicht auf eine Organspende.

Welche dieser Möglichkeiten zur Anwendung kommt, hängt von den Umständen ab. Tritt der Kreislaufstillstand auf dem Weg zum Operationssaal ein, wird die rasche Organentnahme im Operationssaal angestrebt. Findet der Kreislaufstillstand noch auf der Intensivstation statt, sollte entweder der rasche Transport in den Operationssaal erfolgen oder Perfusionssonden eingelegt werden.

\subsection{Therapieabbruch (Maastricht 3)}

Das Vorgehen und der Ort für den Therapieabbruch sowie die anschliessend geplanten medizinischen Massnahmen müssen mit den Angehörigen in Ruhe und detailliert vorbesprochen werden. Es muss primär geklärt werden, bis wann die Angehörigen am Bett des Patienten bleiben wollen. Es ist wichtig, darüber aufzuklären, dass der unmittelbar zum Tod führende anhaltende Kreislaufstillstand häufig sehr rasch, unter Umständen aber auch erst mehrere Stunden nach Therapieabbruch eintreten kann. Die Angehörigen müssen wissen, dass nach Eintritt des Kreislaufstillstands und festgestelltem Tod wegen der organschädigenden warmen Ischämie die Organentnahme so rasch wie möglich erfolgen muss. Sie müssen auf den Zeitdruck vorbereitet sein, der besteht, nachdem der Herzstillstand eingetreten ist, und sie sollten die Möglichkeit haben, sich vorher vom sterbenden Patienten zu verabschieden.

Wird der Therapieabbruch auf der Intensivstation durchgeführt, besteht einerseits die Möglichkeit, nach Feststellung des Todes rasch in den Operationssaal zu fahren, um dort die Organe zu entnehmen. Dieses Vorgehen ist nur für die weniger ischämieanfälligen Organe wie zum Beispiel die Nieren oder auch die Lunge möglich. Die Angehörigen müssen auf den Zeitdruck und die rasche Verlegung des ver- storbenen Spenders vorbereitet werden. Andererseits besteht auch die Möglichkeit, nach Feststellung des Todes die femoralen Gefässe des Spenders auf der Intensivstation zu kanülieren und dort mit der Perfusion der Organe zu beginnen (vgl. Kapitel 5.3.).

Mit den Angehörigen muss insbesondere auch über den Ort des Therapieabbruches gesprochen werden. Wenn sie der Organentnahme (vor allem im Hinblick auf die Transplantation besonders ischämieanfälliger Organe wie der Leber) im Operationssaal zustimmen, ist es grundsätzlich möglich, dass sie den sterbenden Patienten mit in den Operationssaal begleiten und bei ihm bleiben, bis der Kreislaufstillstand eintritt. Dies muss jedoch vorher besprochen sein. Wird als Ort für den Therapieabbruch der Operationssaal in Betracht gezogen, müssen folgende Punkte beachtet werden:

- Die Trennung der Prozesse des Therapieabbruchs und der Organentnahme muss unter erschwerten Umständen aufrechterhalten werden;

- Während des Therapieabbruchs bis zur Todesfeststellung orientiert sich die palliative Behandlung ausschliesslich am Wohl des Patienten;

- Verzögert sich der Eintritt des Todes so lange, dass eine Entnahme der Organe in optimalem Zustand gefährdet erscheint, darf kein Druck auf den behandelnden Arzt entstehen, den Tod zu beschleunigen;

- Die besonderen Umstände an diesem Ort des Therapieabbruchs müssen vorgängig mit den Angehörigen besprochen werden.

Die Angehörigen müssen darüber aufgeklärt werden, dass die Organentnahme möglicherweise nicht stattfinden kann, wenn der Kreislaufstillstand erst nach einer langen Periode mit sehr tiefem Blutdruck und entsprechend langanhaltender, ungenügender Blutund Sauerstoffversorgung der Organe eintritt.

\subsection{Medizinische Massnahmen}

Massnahmen zur Aufrechterhaltung der Organperfusion wie Herzmassage (Kardiokompression) oder Einlegen von femoralen Kanülen zur kalten Organperfusion erfolgen bei Spendern der Kategorie 1, 2 und 4, nachdem der Tod festgestellt worden ist. Wenn der Spender selbst keine Einwilligung zur Organspende erteilt hat, dürfen zur Überbrückung der Zeit bis zum Vorliegen der Einwilligung der Angehörigen organerhaltende medizinische Massnahmen längstens über 72 Stunden durchgeführt werden [23]. Haben die Angehörigen nach Ablauf dieser Zeitspanne keine Zustimmung zur Organentnahme erteilt, ist eine Organentnahme ausgeschlossen.

Die Frage nach der Durchführung von medizinischen Massnahmen vor der Feststellung des Todes stellt sich ausschliesslich bei allfälligen Spendern der Kategorie 3. Bei dieser Patientengruppe ist der Therapieabbruch geplant, und vorbereitende medizinische Massnahmen zur Erhaltung der Qualität der Organe und zur Verkürzung der warmen Ischämiezeit sowie 
serologische und immunologische Tests können eingeleitet werden, sobald die Voraussetzungen gemäss Kapitel 3.2. erfüllt sind.

Es handelt sich insbesondere um die folgenden Massnahmen:

a) Verabreichung von gerinnungshemmenden Medikamenten: Gerinnungshemmende Medikamente werden unmittelbar vor dem Kreislaufstillstand verabreicht, also zu einem Zeitpunkt, in welchem der Tod unmittelbar bevorsteht.

b) Einlegen von Perfusionssonden: Das Einlegen von Perfusionssonden erlaubt eine rasche Perfusion der Organe nach Eintritt des Kreislaufstillstands und optimiert die Funktionsfähigkeit der zu entnehmenden Organe. Damit kann ein hastiger Transport des Spenders in den Operationssaal vermieden werden.

\section{Betreuung der Angehörigen}

Der Tod infolge primärer Hirnschädigung ist schwerer zu begreifen als der Tod durch einen Kreislaufstillstand. Der an einer primären Hirnschädigung verstorbene Patient wirkt, solange er noch beatmet wird, auf die meisten Menschen nicht wie ein Toter im herkömmlichen Sinn; der Brustkorb hebt und senkt sich durch die Beatmungsmaschine, die Haut ist warm, der Puls ist tastbar und manchmal können äussere Reize sogar - durch Rückenmarkreflexe vermittelte Bewegungen und Kreislaufreaktionen auslösen. Dies führt immer wieder zu Verunsicherungen. In diesem Moment des Abschiednehmens wird das an die Angehörigen herangetragene Ersuchen, sich innert kurzer Zeit zum mutmasslichen Spendewillen des Verstorbenen zu äussern, häufig als zusätzliche Belastung erlebt [24]. Noch grösser ist der Zeitdruck bei der NonHeart-Beating-Organspende (Maastricht-Kriterien 1, 2 und 4). Andererseits kann die Aussicht, dass die Organe des Verstorbenen anderen Menschen das Leben retten oder verbessern können, auch als Trost empfunden werden.

Angehörige haben ein grosses Bedürfnis nach Informationen, die ein gewisses Mass an Entlastung bieten können. Die lokale Transplantationskoordination nimmt in dieser Situation als prozessübergreifende Instanz eine Sonderstellung ein. Sie trägt deshalb auch die Verantwortung, dass der Organspendeprozess sowie der Informationsfluss optimal laufen und koordiniert allenfalls weitere Massnahmen im Bereich der Angehörigenbetreuung.

Die Angehörigen sollen über alle wesentlichen Schritte informiert sein. Sie sollten im Besitz einer Kontaktadresse sein, die kompetente Hilfestellung bietet oder die Angehörigen an die richtige Stelle weiterleiten kann. Sie müssen insbesondere wissen, unter welchen Umständen und zu welchem Zeitpunkt sie sich vom Verstorbenen verabschieden können. Es bedarf grosser Erfahrung und Empathie, um auch nicht ausgesprochene Bedürfnisse zu erkennen, gerade angesichts des Zeitdrucks. Die Rollenverteilun- gen im Organspendeprozess sowie die Aufgaben, Kompetenzen und Verantwortlichkeiten der involvierten Personen müssen definiert sein.

\section{Umgang mit dem Leichnam}

Dem Leichnam des Organspenders ist vor, während und nach der Organentnahme mit demselben Respekt und unter denselben Vorgaben zu begegnen, wie dies mit jeder frisch verstorbenen Person der Fall ist. Der Leichnam des Organspenders muss den Angehörigen in würdigem Zustand zur Bestattung übergeben werden. Die lokale Transplantationskoordination ist dafür verantwortlich sicherzustellen, dass die Angehörigen über alle relevanten Informationen (insbesondere auch über allfällige zeitliche Verzögerungen, z. B. bei rechtsmedizinischen Abklärungen) verfügen.

Bei der Organentnahme hat man es mit dem Körper eines Toten zu tun. Dieser besitzt jedoch nach wie vor ein weitgehend funktionstüchtiges spinales und autonomes Nervensystem (vgl. III. Anhang; A. Klinische Zeichen des Todes; Absatz 5). Der tote Körper kann deshalb auf Reize reagieren und motorische Reaktionen zeigen. Mit der Verabreichung von Anästhetika können solche Reaktionen weitestgehend verhindert werden. Dies trägt zur Entlastung der bei einer Organentnahme involvierten Personen bei. Da die Verabreichung von Anästhetika bis zu einem gewissen Grade ischämieprotektiv wirkt und einer Verletzung der zu entnehmenden Organe vorbeugt, ist sie auch im Interesse des Empfängers. Aus diesen Gründen wird die Verabreichung von Inhalationsanästhetika empfohlen.

\section{Schulung und Unterstützung des Behandlungsteams}

Pflegefachpersonen sowie Ärzte in Intensivstationen sind in ihrem Alltag psychisch belastenden Ereignissen ausgesetzt. Die Betreuung eines Organspenders ist für das Behandlungsteam der Intensivstation eine grosse Herausforderung. Das persönliche Erleben, der Umgang mit solchen Situationen und ihre Bewältigung sind von Mensch zu Mensch unterschiedlich.

Die Verarbeitung des Schicksals des Verstorbenen und seiner Angehörigen sowie die verschiedenen Interaktionen zwischen behandelnden Ärzten, Pflegefachpersonen, den Konsiliarärzten, Angehörigen und der Transplantationskoordination fordern ein hohes Mass an fachlichen, psychologischen, kommunikativen und organisatorischen Fähigkeiten und Fertigkeiten und können die Mitglieder des Behandlungsteams an ihre Grenzen bringen.

Wiederholte Informationsveranstaltungen und Schulungen für das Behandlungsteam sind somit unabdingbar. Alle Mitglieder sollten regelmässig in folgenden Bereichen geschult werden:

- Todesfeststellung gemäss den Richtlinien;

- Pathophysiologie des Todes;

- organisatorische Abläufe vor, während und nach einer Organspende; 
- ethische Aspekte der Organspende und des Hirntodkonzeptes;

- Umgang mit den Angehörigen.

Es sollte die Möglichkeit bestehen, schwierige Situationen im Rahmen einer retrospektiven Fallbesprechung mit allen Beteiligten aufzuarbeiten. Alle Intensivstationen, die Organspender betreuen, sollten über Angebote zur Supervision und Stressbewältigung verfügen

\section{Anhang}

\section{A. Klinische Zeichen des Todes}

Der klinischen Untersuchung zur Feststellung der Zeichen des Ausfalls der Hirnfunktionen kommt bei der Feststellung des Todes zentrale Bedeutung zu. Die Umstände und Modalitäten ihrer Durchführung werden im Folgenden beschrieben:

1. Koma bekannter Ursache: Ein Koma bekannter Ursache liegt vor, wenn der Zustand durch den Nachweis struktureller Hirnläsionen mittels Bildgebung hinreichend erklärt ist.

Voraussetzung ist, dass die Kerntemperatur über $35{ }^{\circ} \mathrm{C}$ liegt, kein Schockzustand besteht und der Zustand nicht durch metabolische Störungen oder medikamentöse bzw. toxische Einflüsse mitverursacht ist. In allen anderen Situationen ist eine technische Zusatzuntersuchung notwendig.

2. Beidseits weite, auf Licht nicht reagierende Pupillen: Unvollständig dilatierte oder anisokore Pupillen schliessen die Feststellung des Todes nicht aus, vorausgesetzt, dass sie auf Lichtreize nicht reagieren.

3. Fehlen der okulozephalen (= zervikookulären und vestibulookulären) Reflexe: Wenn bei rascher passiver Kopfrotation sowie Kopfextension und Flexion keine Augenbewegungen auftreten, fehlen die okulozephalen Reflexe. Diese Untersuchung darf nur gemacht werden, wenn ein Trauma der Halswirbelsäule ausgeschlossen ist. Der vestibulookuläre Reflex wird kalorisch (mit Eiswasser) geprüft.

4. Fehlen der Kornealreflexe: Die Kornealreflexe können durch Berührung der Kornea mit einem kompakten Watteköpfchen geprüft werden.

5. Fehlen jeglicher Reaktion auf starken Schmerzreiz: Die Reaktion auf Schmerzreize muss durch Druck auf die Austrittsstelle eines Trigeminusastes am Orbitarand geprüft werden. Gelegentlich persistiert nämlich eine Reaktion der oberen oder unteren Extremitäten auf schmerzhafte Reize, die ausserhalb des Trigeminusgebietes gesetzt werden. Es ist möglich, dass bei einem toten Patienten noch spinale Reflexe mit komplexen motorischen Reaktionen vorhanden sind. Spontane und reflektorische Bewegungen auf Reize schliessen den Hirntod somit nicht aus (wie z.B. Abdominalreflex; Kremasterreflexe; isolierte Zuckungen an den oberen Extremitäten; unilaterale ExtensionsPronationsbewegungen). Im Zweifelsfall ist eine technische Zusatzuntersuchung durchzuführen (vorzugsweise eine nichtinvasive transkranielle Doppler- oder Farbduplex-Sonographie) [25].

6. Fehlen des Husten- und Schluckreflexes: Hustenund Schluckreflexe löst der begutachtende Arzt durch Stimulation der Rachenhinterwand und der Trachealschleimhaut aus.

7. Fehlen der Spontanatmung: Das Fehlen der Spontanatmung muss durch einen Apnoetest belegt werden.

Die Durchführung eines Apnoetestes setzt eine normale neuromuskuläre Funktion voraus. Wenn ein Patient Myorelaxantien bekommen hat, muss eine erhaltene neuromuskuläre Funktion durch neuromuskuläres Monitoring belegt werden.

Der Apnoetest wird in folgenden Schritten durchgeführt:

- Arterielle Blutgasanalyse zur Messung des Ausgangswertes von $\mathrm{PaCO}_{2}$, arteriellem $\mathrm{pH}$ und zur Erstellung der Korrelation zwischen $\mathrm{PaCO}_{2}$ und endtidal $\mathrm{CO}_{2}$;

- Beatmung während 10 Minuten mit 100\% Sauerstoff;

- Kontinuierliche Überwachung mittels transkutaner $\mathrm{O}_{2}$-Sättigungs-Messung;

- Hypoventilation unter endtidal $\mathrm{CO}_{2}$ - oder transkutaner $\mathrm{pCO}_{2}$-Kontrolle mit 0,5-2 1/min, bis ein $\mathrm{PaCO}_{2}$ von $60-70 \mathrm{~mm} \mathrm{Hg}(8-9,35 \mathrm{kPa})$ erwartet werden kann;

- Abnahme einer ABGA zum Nachweis, dass der $\mathrm{PaCO}_{2}$-Partialdruck über $60 \mathrm{~mm} \mathrm{Hg}$ bzw. 8 kPa gestiegen und der $\mathrm{pH}$-Wert unter 7,30 gesunken ist;

- Dekonnektion des Patienten vom Respirator. Die Sauerstoffversorgung wird durch eine Sonde im Trachealtubus mit kontinuierlichem $\mathrm{O}_{2}$-Fluss von 2 bis 4 Litern pro Minute gewährleistet (Kinder mit englumigem Tubus max. 2 L); um ein Barotrauma zu vermeiden, darf die Sonde nicht zu tief liegen;

- Beobachtung des Fehlens von Atembewegungen während 1 Minute;

- Wiederanschluss des Patienten an den Respirator mit den vorgängigen Ventilationsparametern.

Wenn die transkutane $\mathrm{O}_{2}$-Sättigung unter $80 \%$ fällt, ist der Apnoetest vorzeitig abzubrechen.

Bei Patienten mit schweren Oxygenationsstörungen oder relevanter Linksherzinsuffizienz wird zur Vermeidung von gefährlichen Kreislaufstörungen wie oben verfahren, jedoch nicht dekonnektiert:

- Nach Abnahme des ABGA wird das Beatmungsgerät auf einen Spontanatmungsmodus umgestellt (CPAP-Modus ohne Atemassistenz), die Apnoeventilation ausgeschaltet und die Einstellungen weiter so modifiziert, dass spontane Atembewegungen erkannt werden können. Es ist zu bedenken, dass durch zu empfindlich eingestellte FlowTrigger kardial bedingte Atemzüge ausgelöst werden können.

- Beobachtung des Fehlens von Atembewegungen;

- Wiederaufnahme der Beatmung mit den vorgängigen Ventilationsparametern. 
Bei Kindern unter einem Jahr wird unter CPAP am Beatmungsgerät beobachtet und es gilt als Zielwert ein $\mathrm{PaCO}_{2} 90 \mathrm{~mm} \mathrm{Hg}$ (12 kPa [26]) und ein $\mathrm{pH}$-Wert unter 7,25, wobei die Sauerstoffsättigung nicht unter $80 \%$ abfallen sollte.

Wenn der Apnoetest nicht konklusiv durchgeführt werden kann (z.B. bei schwerer chronischer Hyperkapnie), muss analog zur Situation bei nicht beurteilbaren Hirnnerven eine technische Zusatzuntersuchung durchgeführt werden.

\section{B. Technische Zusatzuntersuchungen [27]}

Technische Zusatzuntersuchungen im Rahmen der Feststellung des Todes werden eingesetzt, wenn die Ätiologie des Funktionsausfalls unklar ist. Ziel der technischen Zusatzuntersuchung ist es, den Stillstand der zerebralen Zirkulation zu beweisen.

Die Aussagekraft der technischen Zusatzuntersuchung ist vom arteriellen Mitteldruck während der Untersuchung abhängig. Sie ist gegeben, wenn der arterielle Mitteldruck zum Zeitpunkt der Untersuchung bei Erwachsenen und Kindern mindestens $60 \mathrm{~mm} \mathrm{Hg}$ sowie bei Säuglingen mindestens $45 \mathrm{~mm} \mathrm{Hg}$ beträgt. Der arterielle Mitteldruck zum Zeitpunkt der Zusatzuntersuchung muss zwingend im Untersuchungsbefund festgehalten werden.

Folgende technische Zusatzuntersuchungen sind grundsätzlich geeignet, den Stillstand der zerebralen Zirkulation nachzuweisen:

1. Transkranielle Doppler- oder Farbduplexsonographie;

2. Computertomographie;

3. Magnetresonanztomographie;

4. Digitale Subtraktionsangiographie.

1. Transkranielle Doppler- oder Farbduplexsonographie

Die Untersuchung umfasst die extrakranielle Dopplersonographie an der mittels B-Bild und Color-Flow dokumentierten Arteria $(=\mathrm{A})$ carotis interna und die transkranielle farbkodierte transtemporale Unter-

Hinweise zur Ausarbeitung dieser Richtlinien Auftrag: Am 6. Februar 2009 hat die Zentrale Ethikkommission der SAMW eine Subkommission mit der Revision der medizin-ethischen Richtlinien «Feststellung des Todes mit Bezug auf Organtransplantation» beauftragt.

Verantwortliche Subkommission: Prof. Jürg Steiger, Basel (Präsident); lic. theol. Settimio Monteverde, MAE, Basel (Vizepräsident); Ursula Hager, MAE, Zürich; Prof. Christian Kind, Präsident ZEK, St. Gallen; Dr. Roger Lussmann, St. Gallen; Prof. Philippe Lyrer-Gaugler, Basel; Prof. Stephan Marsch, Basel; Dr. Luca Martinolli, Bern; Prof. Manuel A. Pascual, Lausanne; Dr. Bruno Regli, Bern; Dr. Peter Rimensberger, Genf; lic. iur. Michelle Salathé, MAE, Basel; Dr. Theodor Weber, Bern; Prof. Markus Weber, Zürich. suchung der A. cerebri media beidseits in einer Beschallungstiefe von 55-65 mm mittels pw-Ableitung der Dopplerflusssignale. Charakteristisch für den Stillstand der zerebralen Zirkulation ist, wenn auf beiden Seiten ein Pendelfluss oder nur niederfrequente Spektren (max. $50 \mathrm{~cm} / \mathrm{s}$, von kurzer Dauer <200 ms) vorliegen.

\section{Computertomographie (CT)}

Die Spiral-CT vor und nach intravenöser Kontrastmittelgabe zur Darstellung und Quantifizierung der zerebralen Perfusion (Perfusion-CT) und zur Darstellung der hirnzuführenden Hals- und der intrakraniellen Gefässe (CT-Angiographie) können einen Stillstand der zerebralen Zirkulation nachweisen.

\section{Magnetresonanztomographie (MR)}

Die MR-Angiographie und die Perfusions-MR nach intravenöser Gabe von Gadolinum als Kontrastmittel können einen zerebralen Kreislaufstillstand nachweisen. Die eingeschränkte Verfügbarkeit, die mögliche Inkompatibilität von am Patienten angebrachten Utensilien (Tubus, Sonden, Kabel usw.) sowie die Kontraindikation der Untersuchung bei metallischen Fremdkörpern am Patienten schränken ihre Anwendung erheblich ein.

\section{Digitale Subtraktionsangiographie (DSA)}

Zum Nachweis eines Stillstandes der zerebralen Zirkulation müssen beide Aae. carotides communes und mindestens die dominante A. vertebralis selektiv katheterisiert werden. Bei Injektion in jeder A. carotis communis muss es zu einer Füllung der A. carotis externa und ihre Äste sowie zur Füllung des zervikalen und allenfalls des intrakraniell-extraduralen Abschnittes der A. carotis interna kommen. Besteht bei der Füllung einer A. vertebralis Verdacht auf Hypoplasie dieses Gefässes, muss zusätzlich die A. vertebralis auf der Gegenseite dargestellt werden. Ein Stillstand der zerebralen Zirkulation und damit der Tod wegen Hirnschädigung gilt als erwiesen, wenn die

Beigezogene Experten: Prof. Olivier Guillod, Neuchâtel; PD Dr. Franz Immer, Bern; PD Dr. Luca Remonda, Aarau; Prof. Maja Steinlin, Bern; Prof. Reto Stocker, Zürich

Vernehmlassung: Die Richtlinien wurden im Februar/März 2011 einer breiten Vernehmlassung unterzogen.

Genehmigung: Die definitive Fassung dieser Richtlinien wurde am 24. Mai 2011 vom Senat der SAMW genehmigt.

Inkraftsetzung: Die Richtlinien treten per 1. September 2011 in Kraft.

Die deutsche Fassung der Richtlinien ist die Stammversion. 
zerebralen (d.h. intrakraniell-intraduralen) Arterien und Venen weder im supra- noch im infratentoriellen Kompartiment angefärbt sind.

\section{Protokolle zur Feststellung des Todes}

\section{Flowchart}

\section{E. Literatur}

(Anhang C bis E nicht abgedruckt)

\section{Referenzen}

1 Bundesgesetz vom 8. Oktober 2004 über die Transplantation von Organen, Geweben und Zellen (SR 810.21).

2 Aus Gründen der leichteren Lesbarkeit gilt in diesem Text die männliche Bezeichnung für beide Geschlechter.

3 Guillod O, Mader M. Vorbereitende medizinische Massnahmen im Hinblick auf eine Organentnahme. Rechtsgutachten zu verschiedenen Fragen im Zusammenhang mit dem Transplantationsgesetz. März 2010 (www.transplantinfo.ch od. bag.admin. ch).

4 Dieses Todeskriterium betrifft nur die Situationen, in denen eine Organspende angestrebt wird. In allen anderen Fällen wird der Tod durch einen Arzt / eine Ärztin nach den allgemeinen Regeln der ärztlichen Fachkunde festgestellt. Hier gilt vor allem der irreversible kardiopulmonale Stillstand, welcher in der Folge zum Tod führt, als Haupt-Todeskriterium.

5 Art. 9 TxG sowie Art. 7 Verordnung zum TxG (nachfolgend:TxV).

6 III. Anhang Ziff. A. Klinische Zeichen des Todes.

7 Die Zuverlässigkeit der klinischen Zeichen ist allgemein anerkannt (vgl. Literatur im Anhang). Es gibt keine Hinweise dafür, dass eine Wiederholung der Untersuchung nach einem Beobachtungsintervall bessere Resultate ergibt, sofern die erste Untersuchung lege artis erfolgt ist.

8 III. Anhang Ziff. B Technische Zusatzuntersuchungen.

9 Die Feststellung der Pulslosigkeit durch Palpation ist erwiesenermassen unzuverlässig und erscheint deshalb zur präzisen Feststellung des Zeitpunktes des Kreislaufstillstandes im Hinblick auf eine anschliessende Organspende ungeeignet. Ebensowenig kann das Elektrokardiogramm (EKG) zur Feststellung des Kreislaufstillstandes verwendet werden, da eine elektrische Herzaktivität ohne mechanische Herzaktion oft noch über längere Zeit nach dem Tod im EKG festgestellt werden kann.

10 Das heisst Kinder, die älter als 28 Lebenstage (resp. 44 Wochen postmenstruelles Alter), aber jünger als 1 Jahr sind.

11 Die Zuverlässigkeit der klinischen Zeichen wie auch der Zusatzuntersuchungen zum Nachweis eines irreversiblen Funktionsausfalls des Gehirns ist für das Säuglingsalter weniger gut erforscht als für spätere Altersstufen. Zudem lässt die Vermutung, dass die Nachgiebigkeit der infantilen Schädelstrukturen eine kurzfristige Reversibilität der druckbedingten
Unterbrechung der zerebralen Durchblutung erlauben könnte, ein obligatorisches Beobachtungsintervall ratsam erscheinen.

12 Das heisst, weniger als 28 Lebenstage resp. weniger als 44 Wochen postmenstruelles Alter.

13 Für Neugeborene gelten die Aussagen für ältere Säuglinge (s. Fussnote 11) in verstärktem Masse. Insbesondere sind die Schädelstrukturen besonders nachgiebig. Das notwendige Beobachtungsintervall zur Dokumentation des irreversiblen Ausfalls der zerebralen Durchblutung konnte bisher nicht zuverlässig definiert werden.

14 Im Erwachsenenbereich gilt dies z.B. für Fachärzte der Neurologie und der Intensivmedizin, im Kinderbereich für Ärzte mit einer Weiterbildung in pädiatrischer Intensivmedizin oder in Neuropädiatrie. Bei diesen Weiterbildungen ist die Durchführung der Hirntoddiagnostik in das Curriculum integriert.

15 Art. 8 TxG und Art. 3 TxV.

16 Bis zum Inkrafttreten des neuen Erwachsenenschutzrechts 2013 haben die Angehörigen bzgl. medizinischer Entscheidungen vor dem Tod eines Patienten keine Vertretungsbefugnis, es sei denn, diese werde ihnen im kantonalen Recht eingeräumt.

17 Gemäss Gutachten (Fn. 3 a.a.O. S. 47-48) steht bei Patienten mit einer aussichtslosen Prognose nicht mehr die Lebensrettung oder Gesundheitswiederherstellung im Vordergrund, sondern das Interesse, «in Würde zu sterben». Gestützt auf diese Interpretation ist die Durchführung von organerhaltenden Massnahmen unter den in den Richtlinien definierten Voraussetzungen juristisch vertretbar.

18 Zum Beispiel ein metastasierender Tumor.

19 Auf eine abschliessende Aufzählung der vorbereitenden medizinischen Massnahmen wird verzichtet, da diese ihre Aktualität verlieren kann.

20 Gemäss Art. 30 TxG besteht eine Pflicht, die Tauglichkeit der spendenden Person zu überprüfen Artikel 31 Abs. 1 TxG enthält eine Verpflichtung sicherzustellen, dass die Organe auf Krankheitserreger oder Hinweise auf solche getestet worden sind.

21 Art. 10 Abs. 3 TxG in Verbindung mit Art. 8 TxV.

22 Bei Patienten mit Unterkühlung muss die Kerntemperatur auf $35^{\circ} \mathrm{C}$ angestiegen sein.

23 Art. 10 Abs. 3 TxG in Verbindung mit Art. 8 TxV.

24 Kesselring A. Kainz M. Kiss A. Traumatic Memories of Relatives Regarding Brain Death, Request for Organ Donation and Interactions with Professionals in the ICU. American Journal of Transplantation 2006.

25 Saposnik G, Basile VS, Young B. Movements in Brain Death: A Systematic Review. Can J Neurol Sci. 2009; 36:154-60.

26 Brill R. Bigos D. Apnea threshold and pediatric brain death. Crit Care Med 2000; 28: 1257.

27 Dieses Kapitel stützt sich unter anderem auf die Empfehlungen der Schweizerischen Gesellschaft für Neuroradiologie zum Einsatz neurologischer Zusatzuntersuchungen im Rahmen der Feststellung des Todes vom Oktober 2010 ab, welche im Hinblick auf die vorliegenden Richtlinien ausgearbeitet wurden. Die Empfehlungen sind abrufbar unter www.swissneuroradiology.ch/index.php/fortbildung. html (Rubrik Lehre/Fortbildung/Dokumente). 